\title{
Giant optical activity in dielectric planar metamaterials with two-dimensional chirality
}

\author{
W Zhang, A Potts and D M Bagnall \\ School of Electronics and Computer Science, University of Southampton, \\ Southampton SO17 1BJ, UK \\ E-mail: wz02r@ecs.soton.ac.uk,.ap@ecs.soton.ac.uk and dmb@ecs.soton.ac.uk
}

Received 31 March 2006, accepted for publication 1 August 2006 Published 23 August 2006

Online at stacks.iop.org/JOptA/8/878

\begin{abstract}
For the first time, all-dielectric planar chiral metamaterials consisting of arrays of silicon nitride gammadions on fused silica substrates have been fabricated, and shown to be capable of inducing large changes to the polarization states of transmitted light in a manner that is dependent on the two-dimensional chirality of the microstructured silicon nitride film. The polarization response is found to reverse for opposite enantiomers, and also for the same enantiomer when it is illuminated from opposite sides of the structure. In addition, the polarization states of the various diffracted beams are found to be non-reversible. These structures therefore appear to display elements of non-reciprocal behaviour. The polarization responses of complementary designs, different chiral geometries and various silicon nitride film thicknesses have also been studied. As a result we conclude that multiple reflections within the patterned silicon nitride layer play an important role in defining the mechanism by which these structures are able to modify the polarization states of diffracted light.
\end{abstract}

Keywords: planar chirality, metamaterials, polarization eigenstates, reciprocity

\section{Introduction}

Recently, a new class of nanostructured optical materials has been proposed: the planar chiral metamaterial (PCM) $[1,2]$. The attraction of these structures is that their geometric structure can be artificially engineered in order to manipulate the polarization states of light diffracted from the surface. To date most of the research in planar chiral metamaterials has concentrated on metallic chiral films fabricated on substrates of high resistivity silicon [3-5]. These structures are therefore only transparent for wavelengths above $1.2 \mu \mathrm{m}$ (i.e. in the infrared) and consequently any investigation of their chiral properties in the visible region of the electromagnetic spectrum must be done in reflection. However, this negates the primary advantage that planar chiral structures have with regard to their more familiar three-dimensional variants.

In contrast to the case of three-dimensional (3D) chirality, planar or two-dimensional (2D) chiral materials exhibit an opposite handedness when viewed from opposite directions, and therefore should induce opposite polarization changes for light transmitted through the structure in opposite directions. This offers the possibility for the design of materials with optical properties that may be non-reciprocal under certain circumstances. In addition the planar nature of these structures means they could be incorporated into thin-film coatings for numerous optical components. However, if these structures are to be used in conventional optical devices, they will need to be constructed primarily from optically transparent and lossless materials such as dielectrics.

It has already been shown that thin-film metallic features that individually or collectively possess the geometric property of $2 \mathrm{D}$ chirality are capable of both rotating the polarization direction of light diffracted from the surface, and inducing ellipticity in the diffracted state, and of doing so in a manner that is sensitive to both the handedness and the magnitude of the surface chirality $[3,4]$. This is not entirely unexpected. 


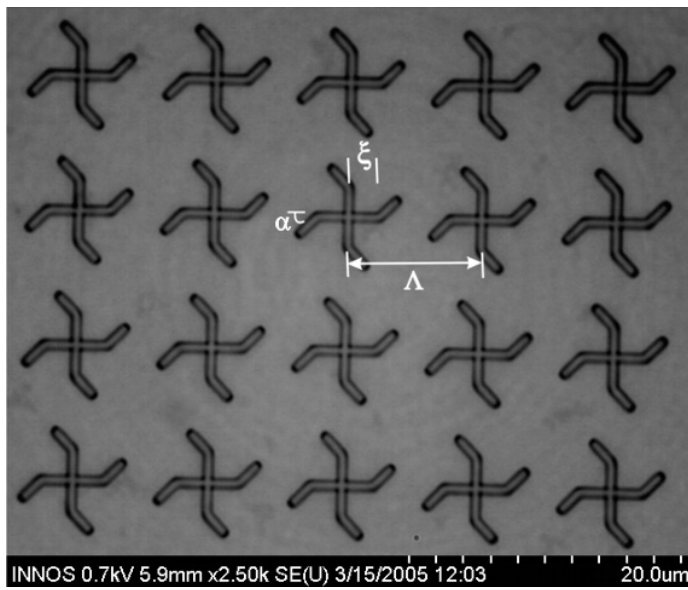

(a)

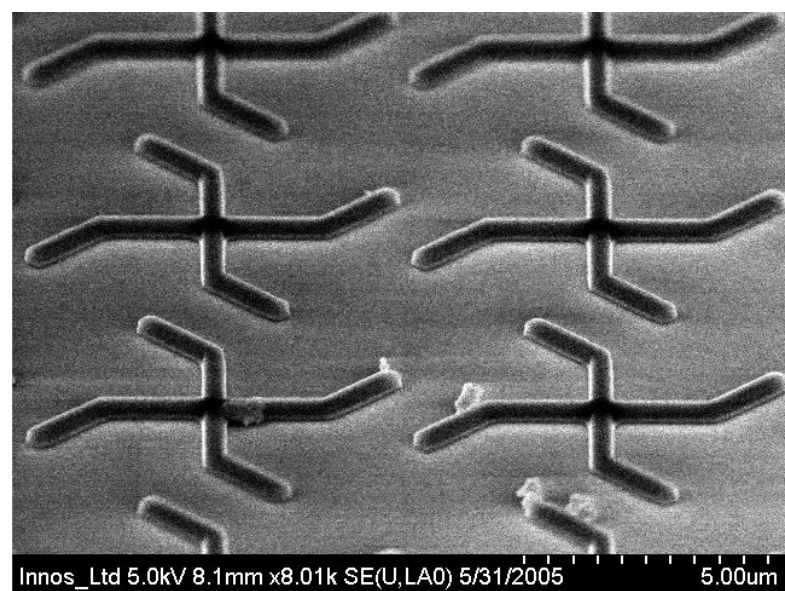

(b)

Figure 1. (a) A scanning electron micrograph of an array of gammadion-shaped holes etched into a thin film of $\mathrm{Si}_{3} \mathrm{~N}_{4}$ on a fused silica substrate. The gammadion bending angle $\left(\alpha=-45^{\circ}\right)$, the pitch $(\Lambda)$, and the characteristic gammadion arm segment length $(\xi)$ are indicated. (b) A scanning electron micrograph of an array of gammadion-shaped $\mathrm{Si}_{3} \mathrm{~N}_{4}$ mesas with $\alpha=-45^{\circ}$ on a fused silica substrate at a tilt angle of approximately $50^{\circ}$.

Various forms of planar chiral behaviour have already been theoretically postulated by a number of authors. Hecht and Barron have suggested that ensembles of planar chiral molecules could exhibit incoherent differential Rayleigh and Raman scattering with circularly polarized light [6], while Arnaut and Davis have calculated the expected scattered fields from a 2D chiral structure formed from a metallic wire gammadion illuminated with microwave radiation and have observed rotations of the polarization azimuth that are dependent on the size, shape and handedness of the chiral elements [7]. More recently Prosvirnin and Zheludev conducted a theoretical analysis of the polarization eigenstates for partial waves diffracted from arrays of planar chiral gammadions [8] and claimed that while the diffraction is compliant with the Lorentz theorem of reciprocity

$$
\int_{S}\left(\mathbf{E}_{1} \times \mathbf{H}_{2}\right)-\left(\mathbf{E}_{2} \times \mathbf{H}_{1}\right) \mathrm{d} S=0
$$

(where $\mathbf{E}_{1}, \mathbf{H}_{1}$ and $\mathbf{E}_{2}, \mathbf{H}_{2}$ are the electric and magnetic fields created by waves propagating in opposite directions [9]), the eigenstates of the array are bi-orthogonal for light diffracted in opposite directions or from opposite enantiomers. Consequently the eigenstates for opposite directions of propagation are not necessarily the same and light propagation along the path of individual diffracted beams may be nonreciprocal.

So far most experimental studies of planar chiral materials have concentrated on the characterization of patterned metallic films and microstructures [3, 4]. However, it has also been demonstrated recently that thin-film polymer layers patterned with 2D chiral structures are also capable of manipulating the polarization state of light [5]. Structures constructed from materials such as dielectrics are especially attractive for device purposes because, unlike their metallic counterparts, they possess no free charge carriers or collective charge excitations (such as excitons and plasmons) that can attenuate the transmitted (or reflected) intensity. If such structures are also fabricated on dielectric substrates such as fused silica, these devices could operate in transmission in the visible region of the electromagnetic spectrum.

In this paper we will present the first ever results of polarization state manipulation by an all-dielectric PCM optical device. These structures all consist of chirally patterned layers of $\mathrm{Si}_{3} \mathrm{~N}_{4}$ on substrates of fused silica. We will show that these structures are capable of generating massive optical activity with specific azimuth rotations in excess of $1000 \mathrm{rad} \mathrm{mm}^{-1}$, and that this optical response is reversed when the sense of chirality of the surface is reversed, thereby indicating that the polarization response is due to microscopic chiral patterning of the sample surface. In addition, the magnitude of the polarization changes is clearly observed to be dependent on the degree of 2D chirality of these patterned layers as well as their thickness. From this latter observation we conclude that the polarization altering mechanism of these structures could be a consequence of quantum interference within a Fabry-Perot cavity formed by the thin (less than $1 \mu \mathrm{m}$ thick) $\mathrm{Si}_{3} \mathrm{~N}_{4}$ layer, and that the presence of such a layer is essential for the chirality-dependent polarization changes to be observed.

\section{Sample fabrication}

The PCM samples were fabricated using a combination of high-resolution electron beam lithography (EBL) and reactive ion etching (RIE). Two different generic designs of planar chiral metamaterial have been studied together with their enantiomeric forms. These designs all consist of gammadions (see figure 1) and are characterized by the differing bending angles $(\alpha)$ of the gammadion leg in each case.

The 2D chiral patterns were all fabricated in $\mathrm{Si}_{3} \mathrm{~N}_{4}$ layers that were supported on $1 \mathrm{~mm}$ thick fused silica (quartz) wafers. Fused silica was chosen because it is transparent at $632 \mathrm{~nm}$ while the fused structure ensured that the substrates were not optically active or birefringent, unlike single-crystal quartz, which is both. 
The wafers were processed using standard microfabrication techniques. First the wafers were cleaned, initially in fuming nitric acid and then with a standard RCA cleaning process, before a $\mathrm{Si}_{3} \mathrm{~N}_{4}$ layer was deposited onto one surface by plasma enhanced chemical vapour deposition (PECVD). Then, following a $2 \mathrm{~h}$ dehydration bake at $140^{\circ} \mathrm{C}$ and exposure to a vapour of HMDS adhesion promoter for around 3 min, a layer of UVIII positive resist (or in some cases UVN30 negative resist) was spun onto the $\mathrm{Si}_{3} \mathrm{~N}_{4}$ coated surface of the wafer. The resist-coated wafer was then baked at $140{ }^{\circ} \mathrm{C}$ for one minute before being patterned with a number of different planar chiral designs using electron beam lithography.

Each chiral design consisted of a square array of gammadions in a planar tiling arrangement of type 442 in the Orbifold notation of wallpaper group symmetries (or $p 4$ in the International Union of Crystallography notation). The area of each array was approximately $1 \mathrm{~mm}^{2}$ and the different gammadion arrays were then arranged on the silicon wafer with a spacing (centre-to-centre) of approximately $2.6 \mathrm{~mm}$ so that the optical characterization of each array could be performed without the probe laser beam impinging on adjacent patterns. Two different gammadion designs were fabricated with bending angles $(\alpha)$ of $45^{\circ}$ and $90^{\circ}$ respectively, together with their enantiomeric forms. In addition, arrays of achiral crosses corresponding to gammadions with bending angles of zero degrees were fabricated in order to provide a direct comparison between chiral and achiral arrays. In this way the effects that arise due to chirality of the patterned surface could be easily identified and distinguished from those effects that may occur due to other asymmetries, such as those inherent in the experimental alignment or those in the samples that are due to fabrication imperfections.

For each gammadion the length of each of the two segments of each of the four arms $(\xi)$ was $1.8 \mu \mathrm{m}$, and the gammadion pitch $(\Lambda)$ was set to be $5 \mu \mathrm{m}$ (see figure 1(a)). In all cases, the designed width of the gammadion arm was set at $200 \mathrm{~nm}$, although the actual width of the final structures was generally slightly larger due to broadening of the lines during both the lithography and reactive ion etching process steps.

After electron beam exposure, the samples underwent an additional post-exposure bake before being developed in Microposit MF322 developer for $60 \mathrm{~s}$ in order to remove the regions of exposed resist. A hard bake was then used to toughen the resist prior to the resist pattern being transferred to the underlying $\mathrm{Si}_{3} \mathrm{~N}_{4}$ layer by reactive ion etching. For each sample the etch depth was chosen to be equal to the thickness of the $\mathrm{Si}_{3} \mathrm{~N}_{4}$ layer so that the underlying fused silica substrate was revealed at the bottom of the etched trenches in the $\mathrm{Si}_{3} \mathrm{~N}_{4}$ layer. The etch gas used was $\mathrm{CHF}_{3}$. Finally the remaining resist was removed using an oxygen plasma prior to the optical characterization of the structures. In the following section a series of optical experiments on these structures will be outlined and their results discussed. It should be noted, however, that for each experiment the design of the particular sample under investigation is defined by its parameters $\alpha, \Lambda$ and $\xi$ as seen by an observer looking directly at the patterned $\mathrm{Si}_{3} \mathrm{~N}_{4}$ surface of the sample (and not through the substrate), even though in some cases the experiment in question may be performed with the incident laser beam striking the reverse side of the sample first (i.e. the bottom of the fused silica substrate) and the sample therefore appearing to the laser beam as having the opposite chiral handedness. The necessity for this strict definition will become apparent when issues of reciprocity and reversibility are investigated later in this paper and the same sample is measured for beams incident in opposite directions.

\section{Experimental results}

Optical experiments were performed to measure the output polarization state of light diffracted from the various arrays of chiral gammadions with differing bending angles and handedness. The light source used was a HeNe laser operating at $632 \mathrm{~nm}$. In all cases the polarization properties of the planar chiral surface were investigated by studying the behaviour of the first-order diffracted beams in the plane of incidence. These beams were generally found to exhibit strong polarization changes and also to have relatively large luminous intensities compared to most higher-order diffracted beams, thus making them easier to measure. While all higher-order beams also appear to show appreciable polarization effects, in contrast, our studies of the zero-order beam failed to detect any discernable change of polarization, either in reflection or transmission when the samples were illuminated at normal incidence. Previous studies on metallic gammadions $[3,4,10]$ also failed to detect any additional rotation of the zero-order beam in reflection at glazing incidence beyond that which was usually observed for light that undergoes Fresnel reflection from an unpatterned but partially absorbing surface. Although other workers have detected small polarization changes in beams transmitted through non-diffracting arrays of metallic gammadions (where the array pitch $\Lambda$ is significantly smaller than the wavelength of the illuminating radiation, $\lambda$ ), the polarization response in this case exhibited two-fold rotational symmetry as a function of $\phi_{0}$. It was therefore explained by the presence of birefringence due to sample manufacturing errors rather than being a consequence of planar chirality [11].

The experimental arrangement we have used to characterize the polarization properties of our planar chiral structures consisted of a $632 \mathrm{~nm} \mathrm{CW}$ laser that was first passed through a linear polarizer and then through a Fresnel rhomb prism in order to generate a circularly polarized beam. A second linear polarizer was then used to convert the beam back into a linearly polarized state, the azimuthal angle of which $\left(\phi_{0}\right)$ could be rotated radially about the beam direction by rotating the second polarizer. This experimental arrangement therefore allows us to rotate the direction of polarization of the beam incident on the sample without changing the beam's intensity. Finally, in order to ensure that the beam was focused onto a single gammadion array without impinging on any adjacent designs, a plano-convex lens was incorporated into the beam path between the second polarizer and the sample. The long focal length of this lens $(f=20 \mathrm{~cm})$ minimized any possible perturbation of the polarization state of the incident beam as it was transformed from a plane wave into a spherical wave.

After the incident beam hits the chiral samples, diffracted waves propagate in the plane of incidence and at various angles to it, with the scattered light showing a well-defined rectangular diffraction pattern. The sample itself is then rotated about its normal until the two perpendicular axes of the square array of chiral elements are parallel to, and perpendicular to, 


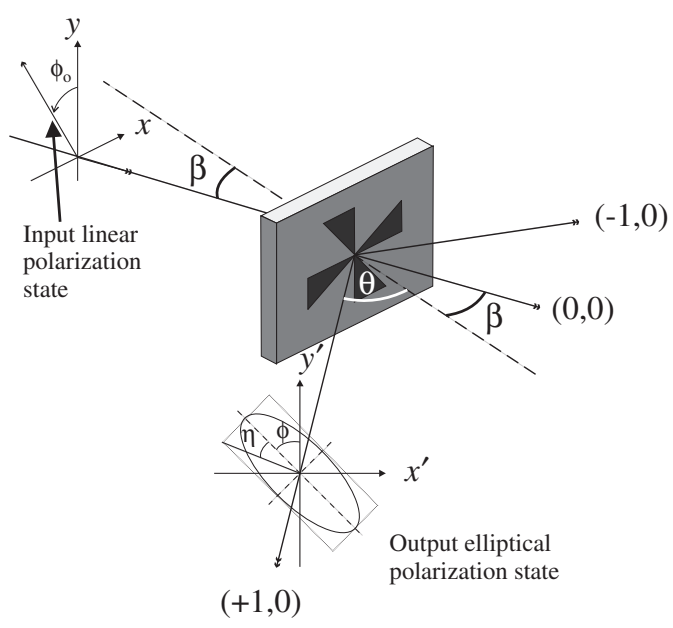

Figure 2. Experimental optical set-up used to characterize the planar chiral structures. The polarization state of each beam is defined by its polarization azimuth $(\phi)$ and ellipticity $(\eta)$, with $\phi$ being measured in an anticlockwise direction from the vertical as viewed by an observer looking directly into the beam. $\phi_{0}$ and $\eta_{0}$ are the equivalent terms for the incident beam. The angle of incidence $(\beta)$ and the diffraction angle $(\theta)$ are measured with respect to the sample normal as indicated. Also shown are some of the diffraction orders.

the plane of the optical bench respectively. The polarization parameters of the first-order diffracted waves in the same horizontal plane as the zero-order beam were then measured and analysed using a commercial polarimeter system (Thorlabs model \#PA510-EC) with a wavelength range of 450-700 nm. The horizontal plane that includes the zero-order beam we will define as being the plane of incidence as it is the plane that includes both the input and directly reflected beams, as well as the normal to the sample. The two first-order beams that lie either side of the zero-order beam in this plane we denote as the $(+1,0)$ and $(-1,0)$ orders respectively.

Most of the results presented here are derived from experiments where the incident light was at normal incidence to the patterned $\mathrm{Si}_{3} \mathrm{~N}_{4}$ surface of the sample. However, the first-order diffracted beams will clearly not be normal to this surface. Consequently, if we were to measure each sample by placing the patterned $\mathrm{Si}_{3} \mathrm{~N}_{4}$ surface of the sample so that it is facing the incident beam, the first- and higher-order diffracted beams on transmission would subsequently strike the back face of the fused silica substrate at an acute angle of incidence. These beams would then undergo additional polarization changes on refraction through this boundary. In order to eliminate this undesired effect, most of our measurements were performed at normal incidence by illuminating the sample from the rear surface first (see figure 2). The incident beam then passes through the silica substrate and only interacts with patterned $\mathrm{Si}_{3} \mathrm{~N}_{4}$ film as it finally leaves the sample. This therefore ensures that the measured polarization changes are due entirely to the interaction of the light beam with the chiral patterned $\mathrm{Si}_{3} \mathrm{~N}_{4}$ layer and are not modified by any subsequent refraction process. The principal exceptions were the experiments on non-reciprocity discussed at the end of this paper.

Figure 2 also clarifies the definitions of both of the polarization parameters $(\phi$ and $\eta)$ for the various beams in our experiments, and the angles used to define their trajectories. In all cases the direction of the incident beam is characterized by the angle of incidence, $\beta$, which we usually measure to the right of the sample normal (for positive values) for an observer looking away from the light source and towards the sample. For most of the measurement reported here, however, the incident light was at normal incidence, so $\beta=0^{\circ}$. The angle of diffraction of the transmitted beam is defined by $\theta$ and is measured to the right of the sample normal for an observer looking through the sample and away from the light source. The diffraction order $(m, 0)$ is then defined by

$$
\sin \left(\theta_{m}\right)+\sin (\beta)=\frac{m \lambda}{\Lambda} .
$$

Diffraction orders for which $m>0$ will therefore be defined as being to the right of the incident beam for this same observer (looking along the incident beam direction away from the source). The polarization states of all beams are then characterized by the parameters $\phi$ (azimuthal angle) and $\eta$ (ellipticity). The azimuthal angle is measured with respect to the vertical direction (or the normal to the plane of incidence) and is defined to be positive for anticlockwise rotations for an observer looking into the beam (see figure 2).

In the following sections we shall report the results of five different experimental investigations of these dielectric planar chiral structures for visible light $(\lambda=632 \mathrm{~nm})$ operating in transmission. These include a comparison of opposite enantiomers and complementary designs, and studies of the effects of gammadion geometry and thickness. The final set of experiments will then address the question of reciprocity in these structures.

\subsection{Enantiomeric symmetry transformations}

Our first experiments were performed at normal incidence $\left(\beta=0^{\circ}\right)$ on samples consisting of arrays of gammadionshaped holes that had been etched into a $320 \mathrm{~nm}$ thick layer of $\mathrm{Si}_{3} \mathrm{~N}_{4}$ on a fused silica substrate. The results presented in figure 3 are for two distinct arrays of gammadion holes with opposite bending angles $\left(\alpha= \pm 45^{\circ}\right)$ but with otherwise identical values for the array pitch $(\Lambda=5.0 \mu \mathrm{m})$, the characteristic arm segment length $(\xi=1.8 \mu \mathrm{m})$ and the line width $(w=200 \mathrm{~nm})$. These two structures therefore represent mirror images of each other. We have investigated the behaviour of these opposing enantiomeric structures for different linearly polarized input states, $\phi_{0}$, and have seen that as the polarization azimuth of the input beam is rotated about the beam direction, the polarization states of both the $(+1,0)$ and the $(-1,0)$ diffraction orders are seen to vary from that of the input beam [12]. In addition, figure 3 shows that not only are strong rotations of the polarization azimuth detected, but significant elliptization of the output beams is also observed. The magnitude of the change to the output polarization azimuth, $\Delta \phi$, and the induced ellipticity, $\eta$, are both strongly dependent on the input polarization azimuth, $\phi_{0}$.

The results presented in figure 3 show two clear trends of behaviour. While identical diffraction orders from opposite chiral enantiomers appear to produce differing optical responses, opposite diffraction orders from the same enantiomer appear to give almost identical responses. This 


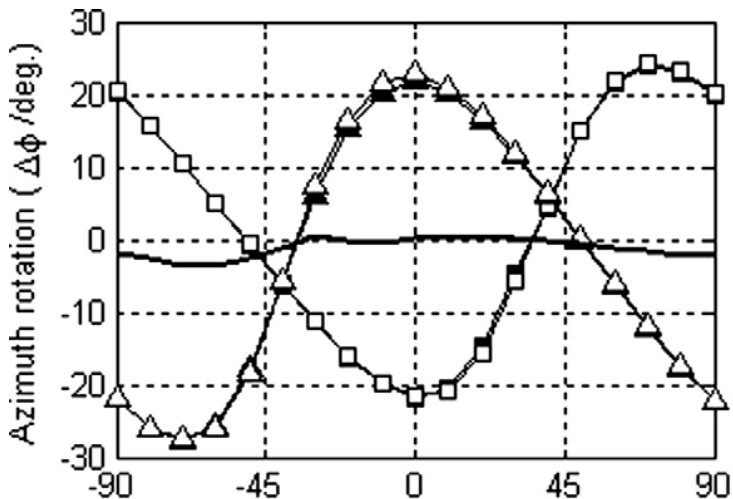

(a) Input polarization azimuth $\left(\phi_{0} /\right.$ deg.)

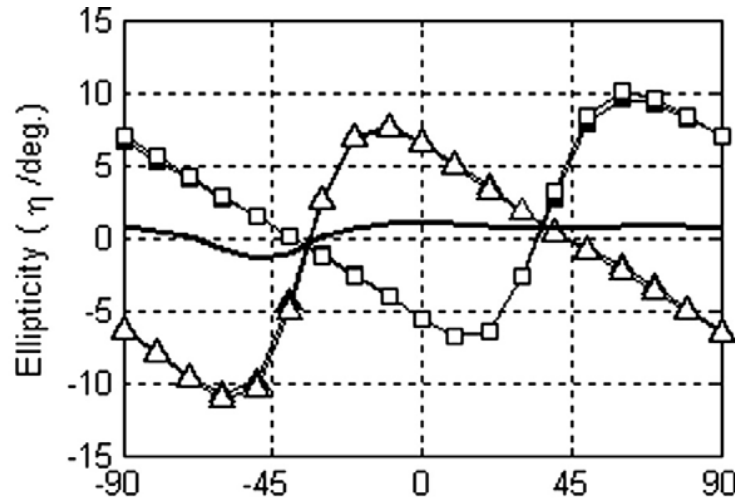

(b) Input polarization azimuth $\left(\phi_{0} / \mathrm{deg}\right.$ )

Figure 3. The polarization change for light diffracted from arrays of gammadion-shaped holes etched in a $320 \mathrm{~nm}$ thick $\mathrm{Si}_{3} \mathrm{~N}_{4}$ film when illuminated with $632 \mathrm{~nm}$ linearly polarized coherent light at normal incidence. (a) Azimuth rotation and (b) ellipticity for the $(+1,0)$ diffraction order from gammadions with $\alpha=-45^{\circ}(\mathbf{\square}),(-1,0)$ order for $\alpha=-45^{\circ}(\square),(+1,0)$ for $\alpha=+45^{\circ}(\mathbf{\Lambda})$, and $(-1,0)$ order for $\alpha=+45^{\circ}(\triangle)$. All arrays have arm segment lengths $\xi=1.8 \mu \mathrm{m}$ and pitches $\Lambda=5.0 \mu \mathrm{m}$. The solid line in (a) represents a plot of equation (5) for the case $(i=1, j=0)$ while that in (b) represents a plot of equation (6) for the same case.

we believe represents significant evidence of the influence of planar chirality in determining the form of the polarization response of these structures. If the behaviour of $\Delta \phi$, and the induced ellipticity, $\eta$, were due to asymmetry in the experimental configuration (such as would be the case if $\beta>$ $0^{\circ}$ ), then opposite diffraction orders might be expected to produce different results from the same sample, while opposite enantiomers would elicit the same response for the same diffraction order. This is not what is observed in figure 3 . Instead the opposite trends are observed. In addition, we have measured the polarization response of a number of different achiral structures formed from arrays of simple crosses etched into $320 \mathrm{~nm}$ thick $\mathrm{Si}_{3} \mathrm{~N}_{4}$ films. These were measured using the same experimental set-up as that used for the gammadions in figure 3 (with an angle of incidence $\beta=0^{\circ}$ ) and yet in this case the first-order diffracted beams in the plane of incidence showed little evidence of either polarization rotation or elliptization. From this we conclude that the polarization changes we see in figure 3 are a direct result of the 2D chirality inherent in the patterned $\mathrm{Si}_{3} \mathrm{~N}_{4}$ surface.

The different polarization changes exhibited by opposite enantiomers can be explained by the application of mirror symmetry (parity) to the experimental configuration for one enantiomer in order to generate a corresponding experimental arrangement for the opposite enantiomeric structure. The theoretical principle underpinning this approach can be found in the expected invariance of Maxwell's equations under parity inversion (P-symmetry), and hence also the invariance of the light-matter interaction under the same transformation. This, however, will only be true if the dielectric and magnetic constants for the material $\left(\epsilon_{r}\right.$ and $\left.\mu_{r}\right)$ are independent of the electric and magnetic field strengths. Crucially, this is usually the case for dielectric materials in weak fields. Thus we should then find that, if the measured polarization change for a diffraction order $(i, j)$ from a left-handed structure is $(\Delta \phi, \eta)$ when that structure is illuminated with linearly polarized light of azimuthal orientation, $\phi_{0}$, then P-symmetry invariance implies that one would expect to measure the opposite polarization change $(-\Delta \phi,-\eta)$ for the opposite diffraction order $(-i,-j)$ for a right-handed structure illuminated with linearly polarized light of the opposite orientation, $-\phi_{0}$ (see figure 4). In terms of the data in figure 3, this implies that the polarization data for opposite diffraction orders from opposite enantiomers can be interchanged simply by rotating one set of data by $180^{\circ}$ about the origin of the graph. We can express this mathematically as

$$
\Delta \phi_{i, j}^{(\mathrm{R})}\left(\phi_{0}, \beta\right)=-\Delta \phi_{-i,-j}^{(\mathrm{L})}\left(-\phi_{0},-\beta\right)
$$

and

$$
\eta_{i, j}^{(\mathrm{R})}\left(\phi_{0}, \beta\right)=-\eta_{-i,-j}^{(\mathrm{L})}\left(-\phi_{0},-\beta\right)
$$

where the superscripts $\mathrm{R}$ and $\mathrm{L}$ refer to right-handed and lefthanded structures respectively. In figure 3 we have tested the accuracy of this interconversion process by plotting the combined functions

$$
\Delta \phi_{i, j}^{(S)}\left(\phi_{0}, 0\right)=\Delta \phi_{i, j}^{(\mathrm{R})}\left(\phi_{0}, 0\right)+\Delta \phi_{-i,-j}^{(\mathrm{L})}\left(-\phi_{0}, 0\right)
$$

and

$$
\eta_{i, j}^{(S)}\left(\phi_{0}, 0\right)=\eta_{i, j}^{(\mathrm{R})}\left(\phi_{0}, 0\right)+\eta_{-i,-j}^{(\mathrm{L})}\left(-\phi_{0}, 0\right)
$$

for each input polarization angle, $\phi_{0}$, for the case $i=1$ and $j=0$. The excellent degree of cancellation of the polarization changes from opposite chiral structures provides further compelling evidence that planar chirality is the primary feature responsible for the observed polarization changes. It also appears to confirm the hypothesis that the light-matter interaction for these structures is a linear effect that is invariant under parity inversion.

The second striking property of the data in figure 3 is the apparent equivalence of opposite diffraction orders from the same enantiomer. At first sight this appears more curious than the behaviour of opposite chiral structures, particularly given that the experimental arrangement in its totality is of mixed symmetry (the sample being asymmetric while the configuration of the opposite diffraction orders is symmetric). This result can be explained though by applying rotational symmetry operations to both the experimental configuration and the input beam independently (see figure 5). 


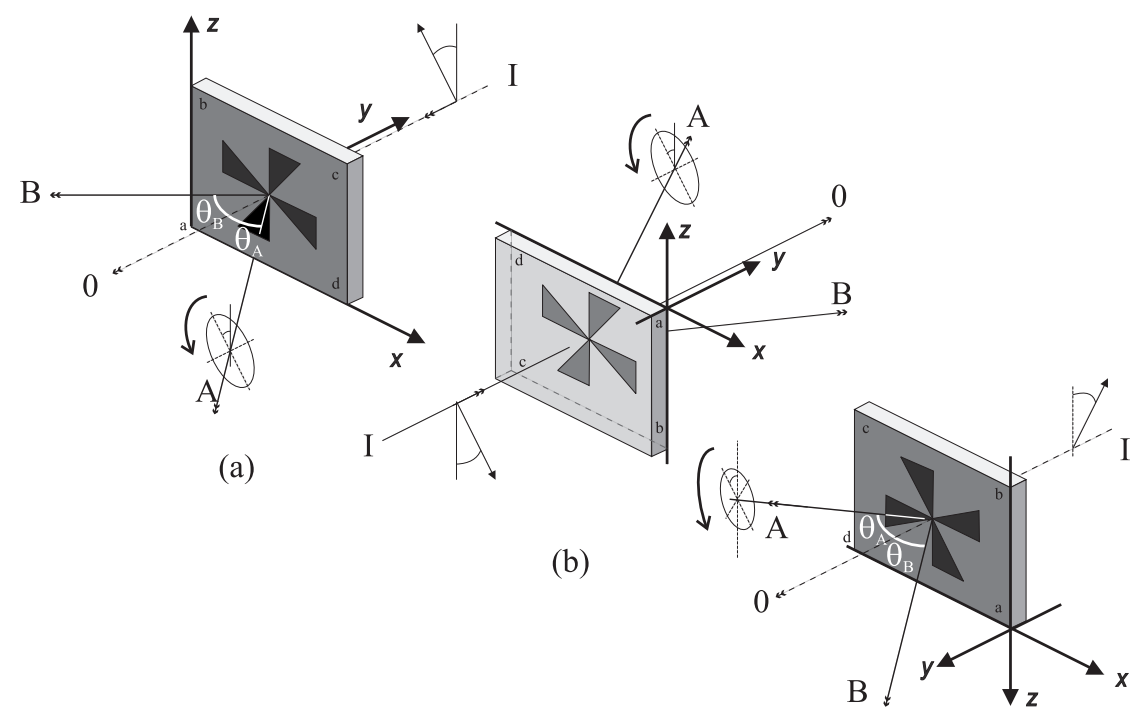

(c)

Figure 4. The effect of a parity transformation on relating the polarization response of opposite chiral enantiomers. (a) Initial experimental arrangement showing the incident beam (I) and various diffracted beams including the zero-order beam (labelled 0). (b) After parity inversion. (c) After a $180^{\circ}$ rotation of the observers viewpoint about the $x$-axis. A comparison of scenarios (a) and (c) indicates that the polarization response of the opposite diffraction order from the opposite enantiomer will be opposite $(-\phi,-\eta)$ for the opposite input state $\left(-\phi_{0}, 0\right)$.
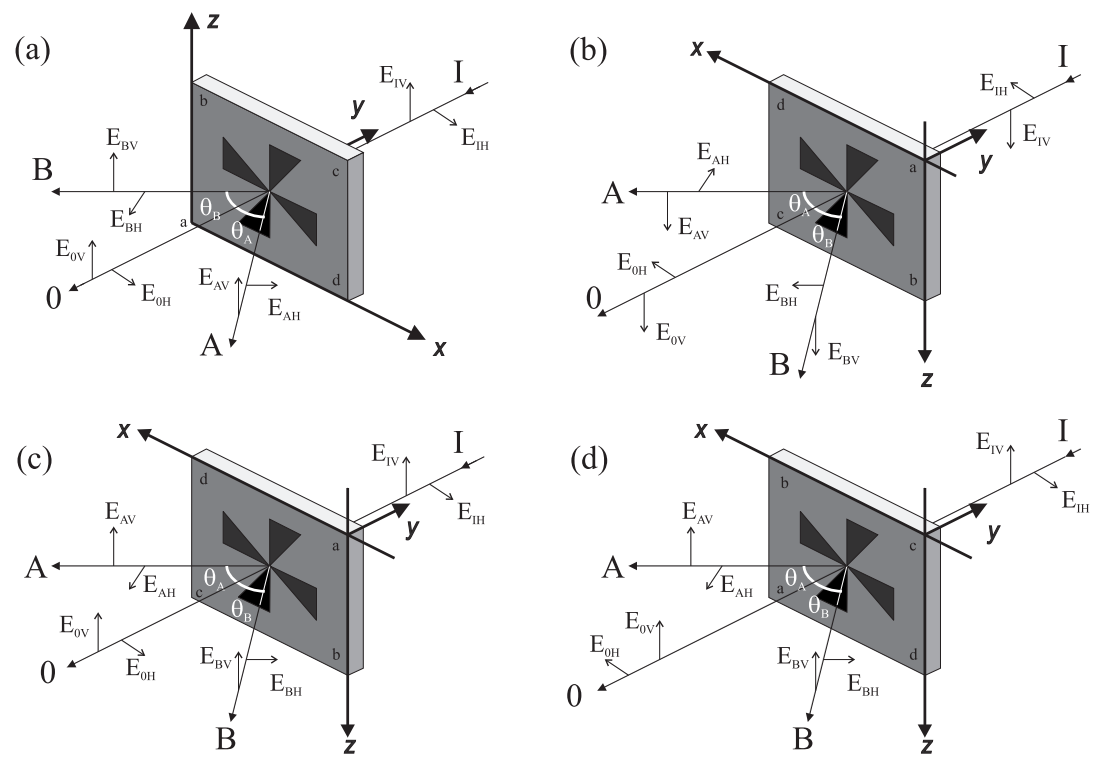

Figure 5. The role of rotational symmetry in defining the equivalence of opposite diffraction orders from a sample possessing two-fold rotational symmetry. (a) Initial experimental arrangement showing the incident beam (I) at normal incidence to the sample and three diffracted beams, A, B and the zero-order beam (0). (b) After a $180^{\circ}$ rotation of the observers viewpoint about an axis normal to the sample. (c) After the input polarization state is rotated by $180^{\circ}$ about the beam axis. (d) After the sample is rotated by $180^{\circ}$ about the beam axis. Note: step (d) is only valid if the sample possesses two-fold rotational symmetry. A comparison of scenarios (a) and (d) indicates that the two scenarios will then be equivalent and so opposite diffraction orders must also be identical.

Because our samples possess four-fold rotational symmetry and the incident beam is normal to the sample, the consequence of these two symmetry rotations is to leave the input configuration of the system unchanged while swapping over the output diffraction orders. This then implies that the output polarization states must also be unaffected by this combination of symmetry operations, and hence the two diffraction orders must have identical polarization states. This is a useful result as it provides a critical test of non-uniformity for both our samples and our experimental set-up. Any discrepancy in the polarization output of the $(-1,0)$ and $(+1,0)$ diffraction orders must be due to sample imperfections or inaccuracies in the optical alignment of the system. Thus such data allow us to estimate the (minimum) experimental error inherent in our system. From the data in figure 3 we conclude that this error is less than $1^{\circ}$ for both $\Delta \phi$, and the induced ellipticity, $\eta$. 
This analysis is of course only valid because our samples have two-fold rotational symmetry (or some multiple thereof) and the incident beam is normal to the sample.

The results we have presented in this section indicate that planar chiral metamaterials are capable of affecting the polarization state of light diffracted by the $2 \mathrm{D}$ chiral topology of the surface. We have seen that these polarization changes are reversed if the sense of chirality of the surface is reversed, thus indicating that it is the $2 \mathrm{D}$ chirality of the surface that is, at the very least, partially responsible for this effect.

\subsection{Complementarity}

In this section, we have compared the properties of complementary structures. This involved fabricating two different chiral samples from identical materials, and patterning the surfaces of the two materials with structures that, if they were to be superimposed, would sum in such a way as to render the resulting combined surface uniformly smooth and featureless. In this instance this meant fabricating one structure that consisted of an array of gammadion-shaped holes in a $\mathrm{Si}_{3} \mathrm{~N}_{4}$ film (the optical properties of such structures were studied in the previous section), and a second structure that consisted of raised gammadion-shaped $\mathrm{Si}_{3} \mathrm{~N}_{4}$ mesas on a silica substrate. For comparative purposes, it was essential that the gammadion features of both structures were identical. This meant fabricating gammadion arrays with the same pitch $(\Lambda)$, gammadion arm segment length $(\xi)$, line width $(w)$ and bending angle $(\alpha)$. Similarly, it was equally essential that the $\mathrm{Si}_{3} \mathrm{~N}_{4}$ layers on both substrates were of equal thickness.

The starting material used for both structures was a $320 \mathrm{~nm}$ thick layer of $\mathrm{Si}_{3} \mathrm{~N}_{4}$ on a fused silica substrate. Both wafers were then patterned with identical designs by electron beam lithography as described in section 2, but for each of the two substrates a different resist layer was used. For one structure (wafer \#3) a $400 \mathrm{~nm}$ thick layer of UVIII was employed. This is a positive resist, and so after development, the area of resist exposed by the electron beam will be removed by the developer solution. For the second structure (wafer \#4), however, a negative resist (UVN30) was used. This was exposed in the same way as the UVIII but with a much lower dose due to its greater sensitivity (approximately 20 times that of UVIII). It was then developed in a 4:1 solution of Microposit MF322 to $\mathrm{H}_{2} \mathrm{O}$. This time the developer removes the unexposed regions and leaves behind the exposed parts of the UVN30 resist. Both wafers then underwent reactive ion etching in $\mathrm{CHF}_{3}$ to a depth of $320 \mathrm{~nm}$ as described in section 2 so that the underlying fused silica substrate is revealed in the regions of the surface where the developer solution had removed the exposed UVIII or unexposed UVN30 resists. After the etching was complete the remaining resist was removed using an oxygen plasma.

As a result of these processes the pattern on wafer \#4 (the UVN30 process) will be the complementary design to that on wafer \#3 (the UVIII process). The former consists of gammadion-shaped mesas of $\mathrm{Si}_{3} \mathrm{~N}_{4}$ on a silica surface while the latter is composed of a continuous $\mathrm{Si}_{3} \mathrm{~N}_{4}$ film into which is etched an array of gammadion-shaped trenches. As the same pattern was used in each case, both gammadion arrays will have the same pitch, $\Lambda$, arm segment length, $\xi$, and bending angle, $\alpha$. The only significant difference will be the linewidth of the gammadions, $w$. While we have attempted to ensure that this is the same for both structures by employing the same design in the electron beam lithography process for both wafers, the different exposure and development conditions of the two resists used inevitably results in some disparity in the final line-width for the two structures. This disparity is then further exacerbated by the reactive ion etching process which is also highly dependent on both the chemical nature of the resist used to mask the substrate and the relative proportions of resist-covered and uncovered areas of the substrate. However, despite these caveats, we have found a compelling degree of similarity in the optical response from each of these structures.

We have characterized and compared both of these structures by again studying the polarization response of the first-order diffracted beams that are transmitted by the sample when it is illuminated at normal incidence. These results are illustrated in figure 6 and clearly show that the polarization response of the $\mathrm{Si}_{3} \mathrm{~N}_{4}$ gammadion mesas on wafer \#4 is virtually identical to that of the gammadion-shaped holes of wafer \#3. This result is a striking manifestation of the principle of superposition as it applies to the far field (Fraunhofer) diffraction regime.

The complementary diffraction screens when added together should generate a non-diffracting unpatterned thin film of uniform thickness. Thus, the diffracted field from one patterned $\mathrm{Si}_{3} \mathrm{~N}_{4}$ screen will cancel with that of its complementary relative (with the exception of the zero-order beams which of course do not cancel). This cancellation can only happen if equivalent diffraction orders from complementary screens have identical amplitudes and polarizations, but opposite phases. This is Babinet's principle. Because it is dependent on the principle of superposition, this implies that the diffraction process for these $\mathrm{Si}_{3} \mathrm{~N}_{4}$ planar chiral structures is linear, and that phase coherence is preserved throughout the process. Given that these structures are designed to be lossless, this is perhaps not surprising. Whether such a situation would still be applicable, however, for planar chiral metallic screens, where it has been suggested [3, 4, 13] that surface plasmons and surface plasmon polaritons (SPPs) may play a significant role in influencing the nature of the light-matter interaction, remains to be tested.

\subsection{Film thickness}

One of the principal attractions of planar chiral metamaterials is their planarity. Not only does this property offer the potential for these structures to be used as optical coatings that could be applied to a large range of existing optical components, it also suggests that such structures could be patterned directly into the existing surfaces of these components without the need for the addition of supplementary thin films that may have other less desirable optical properties. Whether or not this is the case, it is self-evident that the depth to which the chiral pattern is etched, and the choice of material for the patterned surface, should both strongly influence the strength of the resulting polarization effect.

We have already seen that planar chiral metamaterials formed from metallic multilayers are also capable of exhibiting strong polarization effects $[3,4]$ that are similar in form and 


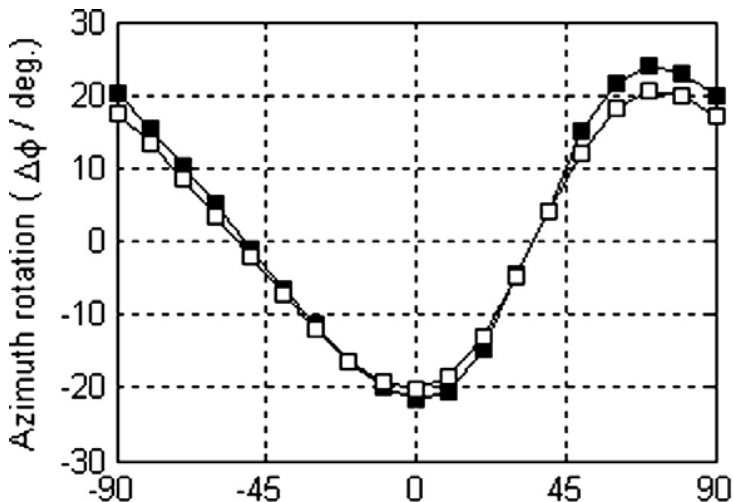

(a) Input polarization azimuth $\left(\phi_{0} /\right.$ deg. $)$

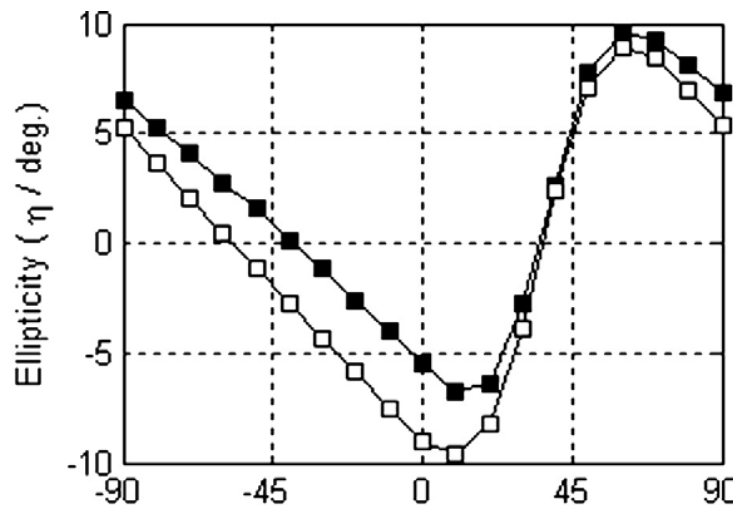

(b) Input polarization azimuth $\left(\phi_{0} / \mathrm{deg}\right)$

Figure 6. A comparison of the polarization changes for the $(+1,0)$ beams diffracted from two complementary planar chiral structures: (i) an array of gammadion-shaped holes ( $\square$ ) etched in a $320 \mathrm{~nm}$ thick $\mathrm{Si}_{3} \mathrm{~N}_{4}$ film on a fused silica substrate, and (ii) an array of $320 \mathrm{~nm}$ high gammadion-shaped $\mathrm{Si}_{3} \mathrm{~N}_{4}$ mesas ( $\square$ ) supported on a fused silica substrate. In each case the gammadion arm segment length is $\xi=1.8 \mu \mathrm{m}$ the bending angle is $\alpha=-45^{\circ}$ and the pitch is $\Lambda=5.0 \mu \mathrm{m}$.

magnitude to those so far presented in this paper, and that a mechanism based on plasmonic excitations has previously been suggested to account for these results $[4,13]$. Clearly though, such a mechanism cannot be present in a dielectric material, and therefore an alternative explanation is required for the polarization properties of these dielectric planar chiral metamaterials. We have attempted to address some of these issues by considering a number of chiral structures that have been etched to a range of different depths, both in thin films of $\mathrm{Si}_{3} \mathrm{~N}_{4}$ on fused silica substrates, and in the uncoated fused silica substrates themselves. Of particular interest were arrays of chiral gammadions etched directly into fused silica to form gammadion-shaped holes or pits.

We considered a range of different etch depths for the chiral patterns etched into the uncoated surface of fused silica wafers: 170, 340 and $690 \mathrm{~nm}$. In addition, six different gammadion sizes as defined by the arm segment length $(\xi)$ were chosen: 1.0, 1.4, 1.8, 2.2, 2.6 and $3.0 \mu \mathrm{m}$. For the three smallest values of $\xi$ the pitch $(\Lambda)$ was set to be $5.0 \mu \mathrm{m}$, while for the three larger values $\Lambda$ was set to be $8.0 \mu \mathrm{m}$. All these structures exhibited well-defined diffraction patterns in both reflection and transmission. However, in contrast to the $\mathrm{Si}_{3} \mathrm{~N}_{4}$ structures of the previous two subsections, none of the $(+1,0)$ and $(-1,0)$ beams diffracted from the patterned silica substrates appeared to show any significant change in their polarization state compared to the input beam when these structures were illuminated at normal incidence. On the other hand, when we fabricated identical arrays of gammadion-shaped holes in various thin films of $\mathrm{Si}_{3} \mathrm{~N}_{4}$, virtually all of the diffracted beams in both reflection and transmission exhibited noticeable polarization changes as a function of input polarization azimuth, $\phi_{0}$. The only exception was the zero-order beam, which, as has been the case for all previous experimental investigations [3-5], showed no polarization effects for normal incidence illumination. This result, however, is consistent with recent theoretical work on the polarization properties of light reflected from structured 2D chiral surfaces [14] where Bedeaux et al have derived modified surface boundary conditions and surface constitutive equations for $2 \mathrm{D}$ chiral interfaces and then shown how they can lead to polarization changes of the reflected light. However, their work also predicts that the polarization change at normal incidence should vanish if the interface structure is isotropic and is invariant under rotations about the normal to the surface. As our structures possess four-fold rotational symmetry, this could explain why the polarization state of the zero-order beam in our experimental arrangement also appears to be unaffected by the surface chirality.

In figure 7 we have presented the results of the polarization change observed for arrays of gammadion-shaped holes etched into three different thicknesses of $\mathrm{Si}_{3} \mathrm{~N}_{4}$ and illuminated at normal incidence at a wavelength of $632 \mathrm{~nm}$. In each case the gammadion pitch $(\Lambda)$ was $5.0 \mu \mathrm{m}$, the characteristic arm segment length $(\xi)$ was $1.8 \mu \mathrm{m}$, the line width $(w)$ was designed to be $200 \mathrm{~nm}$ and the gammadion bending angle $\alpha=-45^{\circ}$. Three different thicknesses of the $\mathrm{Si}_{3} \mathrm{~N}_{4}$ layer were studied: 80,240 and $320 \mathrm{~nm}$; and in each case the etch depth of the gammadion-shaped holes was chosen so that it was equal to the thickness of the $\mathrm{Si}_{3} \mathrm{~N}_{4}$ film used, and thus deep enough to reveal the underlying silica substrate. For all three thicknesses of $\mathrm{Si}_{3} \mathrm{~N}_{4}$, strong polarization changes of the $(+1,0)$ diffracted beam were observed with the magnitude of the polarization change being strongly dependent on the thickness of the $\mathrm{Si}_{3} \mathrm{~N}_{4}$ film. On the other hand, it is also apparent that the input polarization azimuth, $\phi_{0}$, where the polarization change is greatest, appears to remain relatively unchanged as the film thickness is varied.

The results illustrated in figure 7 suggest that the thickness of the $\mathrm{Si}_{3} \mathrm{~N}_{4}$ layer is an important factor in determining the magnitude of the polarization change induced by these chirally patterned films, but plays a significantly smaller role in determining the dependence of the polarization response on the input polarization state. We have also seen that if the $\mathrm{Si}_{3} \mathrm{~N}_{4}$ film is absent and the chiral pattern is etched to a similar depth in the fused silica substrate, no polarization change is detectable at all. From these results we therefore conclude that the presence of a thin surface film (comparable in thickness to the wavelength of the incident radiation) is critical for the operation of these planar chiral metamaterials. As this implies that (a minimum of) two closely spaced interfaces between 


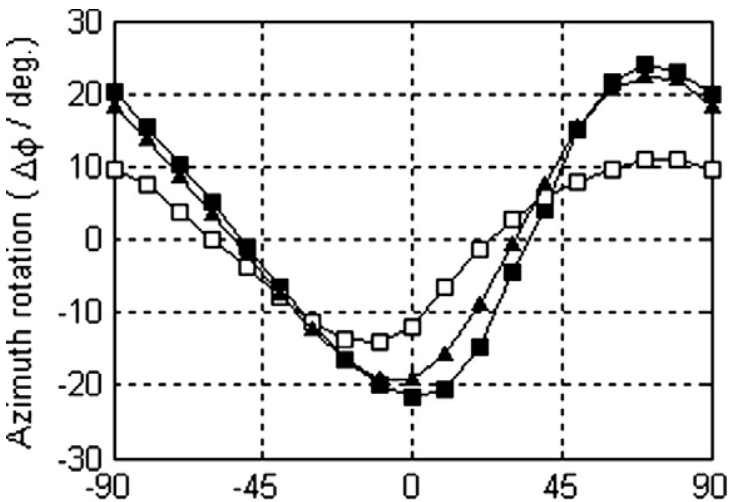

(a) Input polarization azimuth $\left(\phi_{0} / \mathrm{deg}\right.$.)

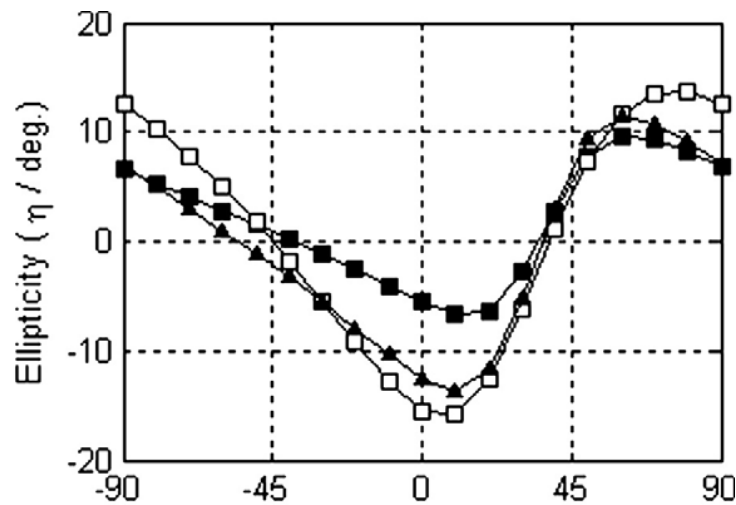

(b) Input polarization azimuth $\left(\phi_{0} /\right.$ deg.)

Figure 7. A comparison of the polarization changes for the $(+1,0)$ beams diffracted from arrays of gammadion-shaped holes etched in $\mathrm{Si}_{3} \mathrm{~N}_{4}$ films of various thicknesses on fused silica substrates. The film thicknesses studied are $320 \mathrm{~nm}(\boldsymbol{\square}), 240 \mathrm{~nm}(\boldsymbol{\Delta})$ and $80 \mathrm{~nm}(\square)$. The gammadions in each array have arm segment lengths $\xi=1.8 \mu \mathrm{m}$, a pitch $\Lambda=5.0 \mu \mathrm{m}$ and bending angles of $\alpha=-45^{\circ}$.

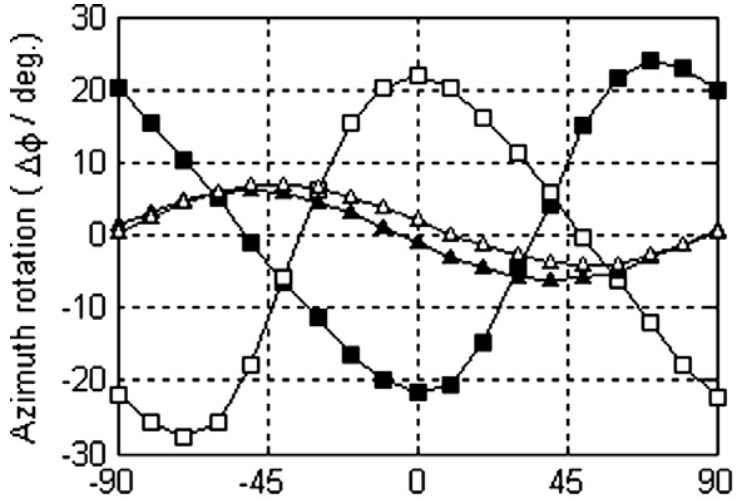

(a) Input polarization azimuth $\left(\phi_{0} /\right.$ deg.)

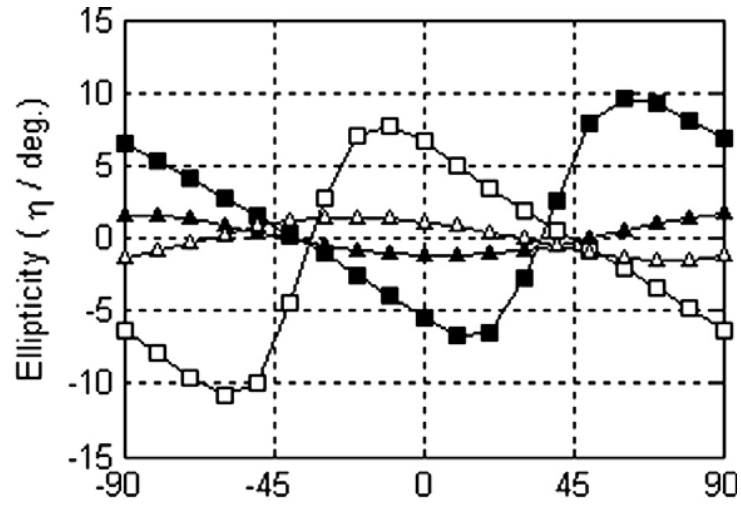

(b) Input polarization azimuth $\left(\phi_{0} /\right.$ deg. $)$

Figure 8. A comparison of the polarization changes for the $(+1,0)$ order beam diffracted from various arrays of gammadion-shaped holes with different bending angles etched in a $320 \mathrm{~nm}$ thick $\mathrm{Si}_{3} \mathrm{~N}_{4}$ film when illuminated with 632 nm linearly polarized coherent light at normal incidence. (a) Azimuth rotation and (b) ellipticity for the $(+1,0)$ diffraction order from gammadions with $\alpha=-45^{\circ}(\square), \alpha=+45^{\circ}(\square)$, $\alpha=-90^{\circ}(\mathbf{\Delta})$, and $\alpha=+90^{\circ}(\triangle)$. All arrays have arm segment lengths $\xi=1.8 \mu \mathrm{m}$ and pitches $\Lambda=5.0 \mu \mathrm{m}$

media of different refractive indices are necessary for these polarization effects to be observed, this in turn suggests that quantum interference in a Fabry-Perot type of optical cavity may be an essential ingredient of these structures, with the etched chiral pattern providing the entry point by which light is coupled into, and diffracted out of, the cavity.

\subsection{Chiral geometry}

In previous papers we have shown that the optical response of a metallic planar chiral surface is dictated at least in part by the shape of the chiral elements on the surface and by their degree of chirality $[4,10]$. It was observed that light rays diffracted from gammadion-shaped holes with bending angles of $\pm 45^{\circ}$ exhibited greater changes to their polarization states than light diffracted from similar sized gammadion features with $\alpha=$ $\pm 90^{\circ}$ and $\alpha= \pm 135^{\circ}$. This appeared to correlate with the predicted chirality index $(K)$ for the respective structures [15] as defined by the function

$K=\frac{1}{4} \int_{\mathbf{r}_{3}} \int_{\mathbf{r}_{2}} \int_{\mathbf{r}_{1}} \rho\left(\mathbf{r}_{1}\right) \rho\left(\mathbf{r}_{2}\right) \rho\left(\mathbf{r}_{3}\right)$

$$
\times \frac{\left|\mathbf{r}_{12}\right|-\left|\mathbf{r}_{13}\right|}{\left|\mathbf{r}_{12}\right|+\left|\mathbf{r}_{13}\right|}\left(\mathbf{r}_{12} \times \mathbf{r}_{13}\right) \mathrm{d}^{2} \mathbf{r}_{1} \mathrm{~d}^{2} \mathbf{r}_{2} \mathrm{~d}^{2} \mathbf{r}_{3}
$$

where

$$
\mathbf{r}_{12}=\mathbf{r}_{1}-\mathbf{r}_{2}
$$

and

$$
\mathbf{r}_{13}=\mathbf{r}_{1}-\mathbf{r}_{3}
$$

and $\rho(\mathbf{r})$ represents the mass distribution function for the $2 \mathrm{D}$ surface. This was despite the fact that the chirality model itself makes no allowance for the optical properties of the material in question. Consequently we were able to assert that the effects we were observing were a direct consequence of the planar chirality of the samples themselves and not due to other factors such as asymmetry in the experimental configuration or the intrinsic optical properties of the constituent materials.

In figure 8 we have presented similar results for gammadion-shaped holes etched in a $320 \mathrm{~nm}$ thick layer of $\mathrm{Si}_{3} \mathrm{~N}_{4}$ on a fused silica substrate. The results illustrated in figures 8(a) and (b) suggest that the optical response from these all-dielectric structures exhibits a similar form of dependence on the gammadion bending angle $(\alpha)$ as was 


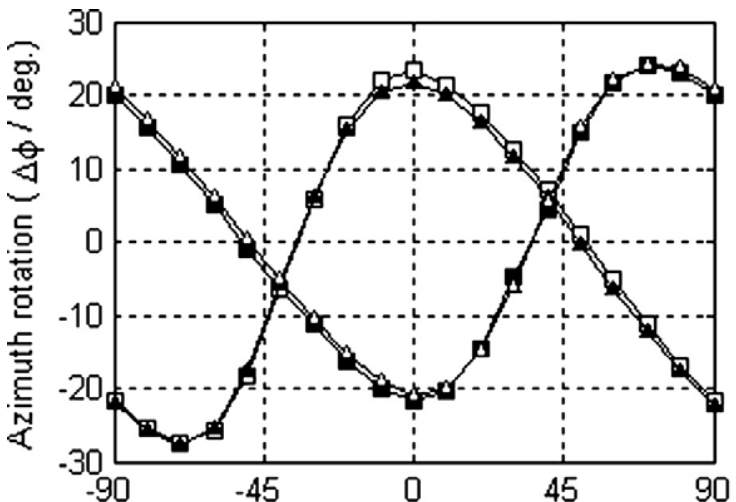

(a) Input polarization azimuth $\left(\phi_{0} /\right.$ deg.)

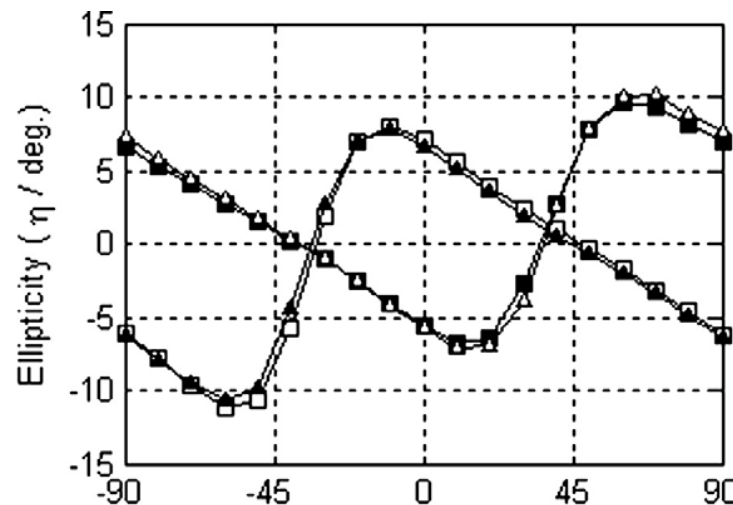

(b) Input polarization azimuth $\left(\phi_{0} / \mathrm{deg}\right.$.)

Figure 9. A comparison of the azimuth rotation (a) and ellipticity (b) for the $(+1,0)$ order beam diffracted from two enantiomer arrays of gammadion-shaped holes with bending angles $\alpha= \pm 45^{\circ}$ etched in a $320 \mathrm{~nm}$ thick $\mathrm{Si}_{3} \mathrm{~N}_{4}$ film when illuminated with $632 \mathrm{~nm}$ linearly polarized coherent light at normal incidence from the front and the back. All arrays have arm lengths $\xi=1.8 \mu \mathrm{m}$ and pitches $\Lambda=5.0 \mu \mathrm{m}$. The various data sets refer to sample W3BA42 $\left(\alpha=-45^{\circ}\right)$ illuminated from the front $\mathrm{Si}_{3} \mathrm{~N}_{4}$ surface ( $\square$ ), sample W3BA42 measured from the substrate side $(\square)$, sample W3BA45 $\left(\alpha=+45^{\circ}\right)$ illuminated from the front $(\triangle)$, and sample W3BA45 illuminated from the substrate $(\boldsymbol{\Delta})$.

observed for the metallic structures studied previously $[4,10]$. The gammadion structures with bending angles of $\alpha= \pm 45^{\circ}$ generate significantly larger changes to the polarization state of the $(+1,0)$ diffracted beams than is seen for structures with $\alpha= \pm 90^{\circ}$.

In addition, it is clear from the data in figure 8 that changes to the bending angle of the gammadions do more than govern the amplitude of the overall polarization changes. They also significantly modify the conditions under which the polarization changes are maximized. In particular, the values of the input polarization azimuth, $\phi_{0}$, at which the greatest change in ellipticity is detected for gammadions with $\alpha=+45^{\circ}$ is significantly different from that seen when $\alpha=+90^{\circ}$. A similar pattern of behaviour is seen for the change in polarization azimuth, $\Delta \phi$. This suggests that the $\phi_{0}$ dependence of the maximum and minimum polarization changes is strongly influenced by the shape of the individual diffracting elements on the surface of the metamaterial (in this case the shape of the gammadions), and hence by the property of 2D chirality. This, therefore, provides further compelling evidence for the proposition that the polarization altering properties of these metamaterials are due in large part to the presence of $2 \mathrm{D}$ chirality.

In conclusion, we have found that there is sufficient experimental evidence from which to conclude that the polarization response from dielectric PCMs is determined not only by the sense of chirality of the surface, but also by the magnitude of the $2 \mathrm{D}$ chirality or the actual topology of the surface.

\subsection{Non-reciprocity and time reversality}

As was pointed out at the start of this paper, the most significant feature of planar chiral metamaterials is the reversal of the sense of chirality for these structures when they are viewed from opposite directions. We have already seen that the chiral patterning of these $\mathrm{Si}_{3} \mathrm{~N}_{4}$ films leads to changes to the polarization states of the transmitted diffracted beams, and that the direction of the polarization change is reversed if both the chirality of the surface and the input polarization state are reversed (by parity inversion). This suggests that the polarization changes manifested by these structures for beams travelling in the reverse direction (where the sense of chirality of the patterned $\mathrm{Si}_{3} \mathrm{~N}_{4}$ films appears reversed) will be opposite and the system will therefore behave non-reciprocally. We have attempted to test this hypothesis by conducting three different experiments.

In the first we have measured the polarization properties of the same structure but from opposite directions (i.e. first with the beam incident normal to the patterned $\mathrm{Si}_{3} \mathrm{~N}_{4}$ surface and then with it incident normal to the substrate side of the sample) as a function of the input azimuth, $\phi_{0}$ (see figure 9). The purpose of this experiment was to test the assertion of Kuwata-Gonokami et al [16] that the polarization changes exhibited by planar chiral layers are due to the presence of residual $3 \mathrm{D}$ chirality rather than $2 \mathrm{D}$ chirality. They studied the polarization altering properties of gold gammadion arrays in transmission in the sub-wavelength regime (where the pitch of the gammadion array $\Lambda$ is less than the optical wavelength $\lambda$ ) and claim to have observed small changes to the polarization state of the transmitted beam which are partially reversed for opposing enantiomeric designs. However, when measured from the reverse direction each of these enantiomeric structures exhibited the same polarization change as it did in the forward direction, thus suggesting that the polarization change was reciprocal and due to 3D chirality. This chirality they attributed to asymmetry in the structure along the direction of light propagation due to an asymmetry in the layer structure of the material and its material interfaces (air-metal-silica-air). In contrast, our results presented in figure 9 indicate that the polarization changes of the first-order diffracted beams are due to $2 \mathrm{D}$ chirality.

We have measured the polarization changes of the $(+1,0)$ diffracted beam from gammadion arrays with $\xi=1.8 \mu \mathrm{m}$, $\Lambda=5.0 \mu \mathrm{m}$ and $\alpha=-45^{\circ}$ (sample W3BA42) and from gammadion arrays with $\xi=1.8 \mu \mathrm{m}, \Lambda=5.0 \mu \mathrm{m}$ and $\alpha=+45^{\circ}$ (sample W3BA45) when each sample is illuminated with linearly polarized light at normal incidence 
from the forward direction (with the laser beam incident on the patterned $\mathrm{Si}_{3} \mathrm{~N}_{4}$ layer first), and at normal incidence from the reverse direction (with the laser beam incident on the silica substrate first). Our results show that the polarization changes of the $(+1,0)$ diffracted beam for sample W3BA42 are parity inverted when the structure is illuminated from opposing directions, and that the polarization change for the reverse direction is almost identical to that of the enantiomeric structure (sample W3BA45 with $\alpha=+45^{\circ}$ ) in the forward direction. We consider this to be unequivocal evidence that the polarization changes manifested by these chirally patterned $\mathrm{Si}_{3} \mathrm{~N}_{4}$ films are due to $2 \mathrm{D}$ chirality. The next question to be addressed is whether these polarization changes are reciprocal.

It should be noted that the concept of reciprocity can be defined in a number of different ways. Suppose we define our initial input state by a Jones vector, $\mathbf{J}(\phi, \eta)$, such that

$$
\mathbf{J}(\phi, \eta)=\left[\begin{array}{l}
p \\
q
\end{array}\right]
$$

where

$$
p=\cos (\phi) \cos (\eta)+\mathrm{i} \sin (\phi) \sin (\eta)
$$

and

$$
q=-\sin (\phi) \cos (\eta)+\mathrm{i} \cos (\phi) \sin (\eta) .
$$

The process for forward propagation through an optical system can be represented as

$$
\mathcal{A} \mathbf{J}\left(\phi_{0}, \eta_{0}\right)=\mu_{a} \mathbf{J}\left(\phi_{1}, \eta_{1}\right)
$$

while that for the reverse direction will be

$$
\mathcal{B} \mathbf{J}\left(\phi_{3}, \eta_{3}\right)=\mu_{b} \mathbf{J}\left(\phi_{2}, \eta_{2}\right) \text {. }
$$

The matrices $\mathcal{A}$ and $\mathcal{B}$ represent the transfer matrices for the respective forward and reverse processes. In general these matrices are not unitary, which is why the complex scaling functions, $\mu_{a}$ and $\mu_{b}$, are included. It should be noted that $\mu_{a}$ and $\mu_{b}$ are functions of the input polarization state in each case, whereas the matrices $\mathcal{A}$ and $\mathcal{B}$ are not. As the Jones vectors are unit vectors, the parameters $\mu_{a}$ and $\mu_{b}$ therefore define the dependence of the output intensity on the input polarization state, a dependence which has been observed and measured previously for planar chiral structures in reflection [17].

A reciprocal system can then be defined as one where, if $\mathbf{J}\left(\phi_{3}, \eta_{3}\right)=\mathbf{J}\left(\phi_{0}, \eta_{0}\right)$, then $\mathbf{J}\left(\phi_{2}, \eta_{2}\right)=\mathbf{J}\left(\phi_{1}, \eta_{1}\right)$. If this is the case then $\mathcal{B}=c \mathcal{A}$ with $c$ being a complex constant, and the system will behave the same when viewed from either side. Our planar chiral systems clearly do not behave in this way as reversing the sample does not yield the same polarization change for a fixed diffraction path. The only exception to this is the zero-order beam which consistently fails to exhibit any polarization change. In this sense our results are entirely consistent with those of Kuwata-Gonokami et al [16] whose experiments on non-diffracting samples are analogous to our experiments on the zero-order beam.

The second form of reciprocity that we could consider is that of reversibility. This can be defined in two ways. The first reverse process involves inputting the output beam of the forward process but along the reverse beam path, and testing to see if the initial state of the forward process is recovered. This process (which we designate the I-process) can be represented as

$$
\mathcal{B} \mathbf{J}\left(\phi_{1}, \eta_{1}\right)=\mu_{b} \mathbf{J}\left(\phi_{2}, \eta_{2}\right) .
$$

The system is reciprocal if $\mathbf{J}\left(\phi_{2}, \eta_{2}\right)=\mathbf{J}\left(\phi_{0}, \eta_{0}\right)$ and $\mathcal{B}=$ $c \mathcal{A}^{-1}$.

The second reverse process is that of time-reversibility, or T-symmetry. When applied to our chiral structures this process (the T-process) can be represented as

$$
\mathcal{B} \mathbf{J}\left(-\phi_{1}, \eta_{1}\right)=\mu_{b} \mathbf{J}\left(\phi_{2}, \eta_{2}\right)
$$

with the input beam $\mathbf{J}\left(-\phi_{1}, \eta_{1}\right)$ again being directed along the reverse beam path to the forward process. In this case reciprocity requires $\mathbf{J}\left(\phi_{2}, \eta_{2}\right)=\mathbf{J}\left(-\phi_{0}, \eta_{0}\right)$. The rationale behind the T-process lies in the (potential) invariance of Maxwell's equations under time-reversality. It should be noted, however, that time-reversality is only strictly applicable if it is applied to the whole system simultaneously (i.e. to all the diffracted beams with the phase of each preserved), and provided that energy is conserved in the forward and reverse processes.

We have tested these reciprocity relations experimentally (see figure 10) by using a combination of a linear polarizer and a quarter wave-plate to create the reverse inputs $\mathbf{J}\left(\phi_{1}, \eta_{1}\right)$ and $\mathbf{J}\left(-\phi_{1}, \eta_{1}\right)$ for each output generated by the forward process in equation (13). The forward experiment was performed by illuminating sample W4BA42 at normal incidence with the linearly polarized incident beam first passing through the fused silica substrate before diffracting from the array of $320 \mathrm{~nm}$ thick $\mathrm{Si}_{3} \mathrm{~N}_{4}$ gammadion mesas on the opposite surface. The polarization state of the $(-1,0)$ diffraction order at a diffraction angle $\theta=-7^{\circ}$ was then measured as a function of the input azimuthal angle, $\phi_{0}$ (see data with black squares in figure 10). The reverse processes were then performed using each of the states $\mathbf{J}\left(\phi_{1}, \eta_{1}\right)$ and $\mathbf{J}\left(-\phi_{1}, \eta_{1}\right)$ as inputs in turn. Each input was incident on the patterned $\mathrm{Si}_{3} \mathrm{~N}_{4}$ surface at an angle of incidence $\beta=7^{\circ}$ (as defined in figure 2). On diffraction from the gammadion array the $(+1,0)$ order beam then passes through the fused silica substrate in a direction normal to, and away from, the patterned $\mathrm{Si}_{3} \mathrm{~N}_{4}$ layer. If the system is reciprocal the resulting $(+1,0)$ order output beam for one or other of the reverse processes should be linearly polarized with an azimuthal angle equal to the original input azimuth $\left(\phi_{0}\right)$ for the I-process, or $-\phi_{0}$ for the T-process.

The data in figure 10 provide clear evidence that the diffraction process for individual beams is in general nonreciprocal as neither the I-process nor the T-process fully recovers the relevant initial input state. If either process were to satisfy the reciprocity requirements outlined above, the final ellipticity should be zero and the final azimuth equal to $+\phi_{0}$ for the I-process or $-\phi_{0}$ for the T-process. While the T-process results in an almost total cancellation of the ellipticity generated by the forward process, the output azimuth deviates significantly from the required linear dependence on $\phi_{0}$. For the I-process neither the azimuth nor the ellipticity fully reverses from that generated by the forward process. From these results we conclude that the polarization changes for the first-order diffracted beams are non-reversible.

It should be noted, however, that while higher-order beams such as the $(+1,0)$ beam appear to produce non-reciprocal 


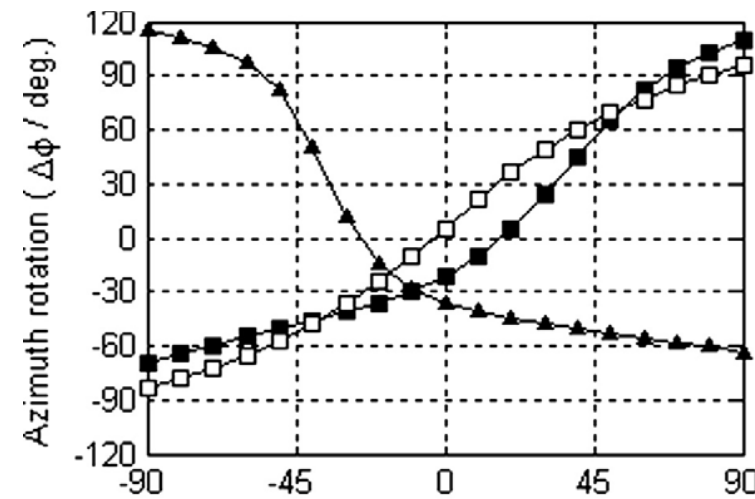

(a) Input polarization azimuth $\left(\phi_{0} / \mathrm{deg}\right.$ )

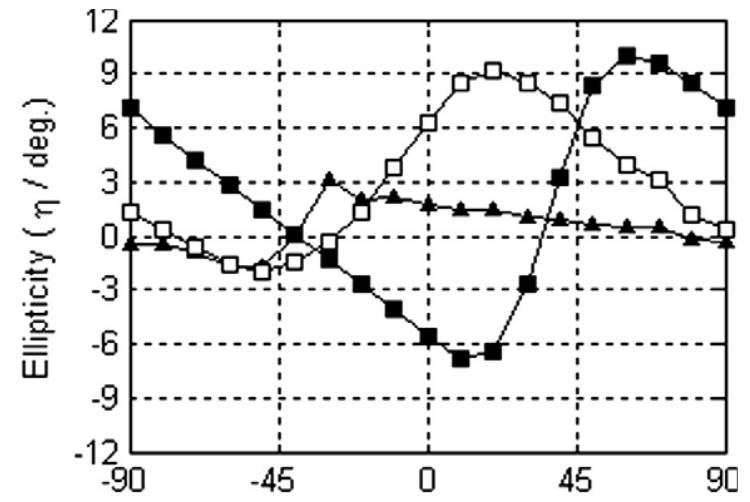

(b) Input polarization azimuth $\left(\phi_{0} / \mathrm{deg}\right.$.)

Figure 10. The azimuthal angle (a) and ellipticity (b) resulting from the I-process $(\square)$ and T-process $(\boldsymbol{\Lambda})$ reciprocity tests for the $(+1,0)$ order beam diffracted from an array of $320 \mathrm{~nm}$ thick $\mathrm{Si}_{3} \mathrm{~N}_{4}$ gammadion-shaped mesas illuminated at an angle of incidence of $\beta=+7^{\circ}$. Also shown for comparison are the azimuth and ellipticity for the $(-1,0)$ beam of the forward process at normal incidence ( $\square$ ). All three data sets are plotted against the azimuthal angle of the linearly polarized input beam for the forward process, $\phi_{0}$. All experiments were performed on the same array of gammadions with arm segment lengths $\xi=1.8 \mu \mathrm{m}$, bending angles $\alpha=-45^{\circ}$ and pitch $\Lambda=5.0 \mu \mathrm{m}$.

(a)

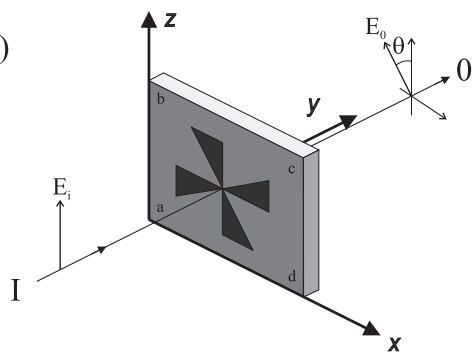

(c)

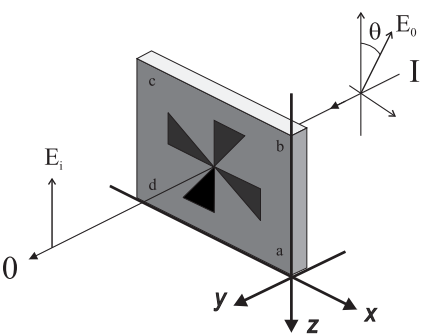

(b)

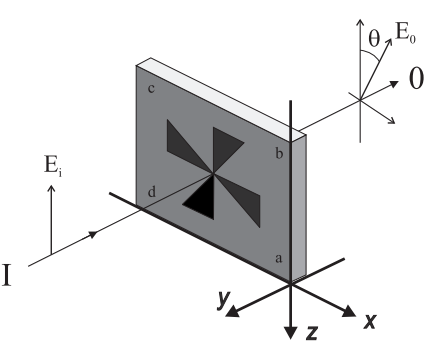

(d)

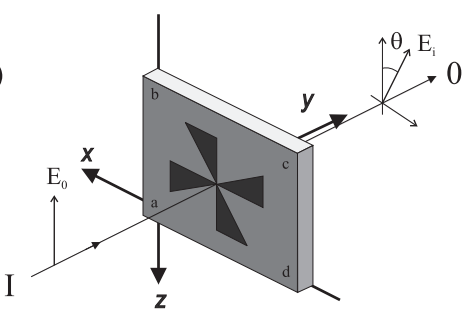

Figure 11. The effects of parity inversion (P-symmetry) and time reversality (T-symmetry) on the polarization state of the zero-order beam at normal incidence. (a) Initial experimental arrangement showing the incident beam (I) at normal incidence to the sample and the transmitted zero-order beam (0). (b) Parity-inverted form of the experimental arrangement in (a). (c) Time-reversed form of the experimental arrangement in (b). (d) After the sample is rotated by $\theta$ about the beam axis. Note: step (d) is only valid if the sample possesses four-fold rotational symmetry. A comparison of scenarios (a) and (d) indicates that the two scenarios are equivalent and so the polarization changes must also be identical.

polarization effects, we have found in all our experiments here and elsewhere $[3,4,10]$ that the zero-order beam consistently fails to exhibit any polarization rotation. The reason for this can perhaps be understood by considering the effect of parity and time-reversal symmetry transformations on an experimental configuration where the only output beam is the undiffracted zero-order beam (see figure 11). The effect of successive parity and time-reversal transformations is to leave the beam incident on the same side of the planar chiral metamaterial (PCM). However, the input is now rotated with respect to the initial input (figure 11(a)) and the polarization change of the output beam is in the opposite direction. If the PCM has four-fold rotational symmetry, the sample can be rotated so that the input in figure $11(\mathrm{c})$ is in the same direction relative to the sample as that in figure 11(a) without altering the output polarization change relative to the input. The result is an input configuration (see figure 11(d)) that is the same as the initial experimental configuration (see figure 11(a)) but which produces the opposite polarization change. This paradox can only be resolved if the polarization change of the zero-order beam is zero. Hence the zero-order beam must be reciprocal. This result relies on the unique symmetry of the zero-order beam and so is not applicable to higher-order beams.

Overall, however, we have demonstrated experimentally that individual diffracted beams from a planar chiral metamaterial exhibit polarization changes that are nonreversible and (with the exception of the zero-order beam) are also non-reciprocal. Crucially, we have presented the first clear empirical evidence that these PCM structures behave differently for light propagating in opposite directions. 


\section{Summary}

The aim of this work has been to investigate the optical properties of planar chiral metamaterials (PCMs) fabricated entirely from dielectric materials, and to compare and contrast their behaviour with that previously reported for metallic PCMs $[3,4,10]$. The main advantage of these all-dielectric PCMs is that they are transparent in the visible region of the electromagnetic spectrum. This allows their optical properties to be studied in transmission rather than the reflection mode used previously for metallic chiral structures on nontransparent silicon substrates $[3,4,10]$. Thus we are able to exploit the key property of these planar chiral metamaterials: their opposite handedness as seen from opposite sides of the structure. By characterizing the polarization states of light rays diffracted in transmission from these dielectric PCMs we have shown that their polarization changing properties are qualitatively very similar to those of metallic PCMs measured in reflection, despite their differing material properties.

The patterned $\mathrm{Si}_{3} \mathrm{~N}_{4}$ films that we have studied have been shown to induce significant rotations in the polarization azimuth for light diffracted from their surface, and also to introduce various degrees of ellipticity. The magnitude and sense of these polarization changes are seen to depend on both the magnitude and sense of chirality of the patterned surface. Opposite enantiomeric structures are seen to produce differing effects for the same vertical input polarization state at normal incidence, while achiral (symmetric) structures produce little discernable polarization change. The effect of changing the magnitude of the chirality of the surface (via the bending angle, $\alpha$, of the individual gammadions) is also seen to impact directly on the polarization change of the various diffracted beams. Together these results provide compelling evidence for the role of surface chirality in mediating these polarization changes.

However, the results presented here have also provided us with new clues regarding the origin of the polarization changes that these PCMs appear to elicit. Previous work on metallic PCMs $[3,4,10]$ has proposed that surface plasmons, induced currents or surface plasmon polaritons may be responsible for mediating the polarization changes. However, these dielectric PCMs possess no free charge carriers. Therefore none of the existing theories can explain the results presented herein. By studying identical chiral patterns etched in $\mathrm{Si}_{3} \mathrm{~N}_{4}$ films of various thicknesses we have seen that the thickness of the $\mathrm{Si}_{3} \mathrm{~N}_{4}$ film plays a crucial role. Moreover, PCMs formed by etching a chiral pattern directly into an uncoated substrate were found to generate no polarization change, even when the surface patterning was identical in its size, geometry and etch depth to that fabricated in the $\mathrm{Si}_{3} \mathrm{~N}_{4}$ films. From this we conclude that the presence of a multiply reflecting surface film (or Fabry-Perot cavity) appears to be critical for the performance of these structures.
We have also shown that complementary structures appear to possess identical optical properties in accordance with Babinet's principle. This suggests that the optical response of these structures is linear and is governed by the principle of superposition. However, we have also shown that the polarization changes for the first-order diffracted beams are generally non-reciprocal, with equivalent diffracted beams producing different polarization changes when the sample is illuminated from the forward and reverse directions. Only the zero-order beam appears to be reciprocal in nature, probably as a consequence of its unique symmetry.

At the present time it is not yet clear if the mechanisms that we have identified for these new all-dielectric PCMs are of relevance for metallic PCMs. There is some evidence that some metallic PCMs such as chiral structures etched in pure gold films do not exhibit the expected polarization behaviour, whereas similar structures in metallic multilayers (Ti/Au/Ti) have shown strong responses $[3,4,10]$. However, this result, if correct, needs to be investigated further by studying alternative metallic thin-film structures and multilayers.

\section{References}

[1] Arnaut L R 1997 J. Electromagn. Wave 111459

[2] Svirko Y, Zheludev N I and Osipov M 2001 Appl. Phys. Lett. 78498

[3] Potts A, Papakostas A, Zheludev N I, Coles H J, Greef R and Bagnall D M 2002 Mater. Res. Soc. Symp. Proc. 722293

[4] Papakostas A, Potts A, Bagnall D M, Prosvirnin S L, Coles H J and Zheludev N I 2003 Phys. Rev. Lett. 90107404

[5] Potts A, Papakostas A, Bagnall D M and Zheludev N I 2004 Microelectron. Eng. 73/74 367

[6] Hecht L and Barron L D 1994 Chem. Phys. Lett. 225525

[7] Arnaut L R and Davies L E 1995 Proc. Int. Conf. on Electromagnetics in Advance Applications Electromagnet Wave pp 381-4

[8] Prosvirnin S L and Zheludev N I 2005 Phys. Rev. E 71037603

[9] Kong J 1986 Electromagnetic Wave Theory (New York: John Wiley)

[10] Potts A, Papakostas A, Zheludev N I, Coles H J, Greef R and Bagnall D M 2003 J. Mater. Sci. Mater. Electron. 14393

[11] Vallius T, Jefimovs K, Turunen J, Vahimaa P and Svirko Y 2003 Appl. Phys. Lett. 83234

[12] Zhang W, Potts A, Bagnall D M and Davidson B R 2006 J. Vac. Sci. Technol. B 241455

[13] Schwanecke A S, Krasavin A, Bagnall D M, Potts A, Zayats A V and Zheludev N I 2003 Phys. Rev. Lett. 91247404

[14] Bedeaux D, Osipov M A and Vlieger J 2004 J. Opt. Soc. Am. A 212431

[15] Potts A, Bagnall D M and Zheludev N I 2004 J. Opt. A: Pure Appl. Opt. 2 193-203

[16] Kuwata-Gonokami M, Saito N, Ino Y, Kauranen M, Jefimovs K, Vallius T, Turunen J and Svirko Y 2005 Phys. Rev. Lett. 95227401

[17] Zhang W, Potts A, Papakostas A and Bagnall D M 2005 Appl. Phys. Lett. 86231905 